\title{
Risk of sexual transmission of human immunodeficiency virus with antiretroviral therapy, suppressed viral load and condom use: a systematic review
}

\author{
Jennifer LeMessurier MD MPH, Gregory Traversy MSc, Olivia Varsaneux MSc, Makenzie Weekes MSc, \\ Marc T. Avey MSc PhD, Oscar Niragira MD, Robert Gervais MD, Gordon Guyatt MD, Rachel Rodin MD MPH
}

Cite as: CMAJ 2018 November 19;190:E1350-60. doi: 10.1503/cmaj.180311

See related article at www.cmaj.ca/lookup/doi/10.1503/cmaj.181405

\begin{abstract}
BACKGROUND: The Public Health Agency of Canada reviewed sexual transmission of HIV between serodiscordant partners to support examination of the criminal justice system response to HIV nondisclosure by the Department of Justice of Canada. We sought to determine HIV transmission risk when an HIV-positive partner takes antiretroviral therapy, has a suppressed viral load or uses condoms.
\end{abstract}

METHODS: We conducted an overview and systematic review update by searching MEDLINE and other databases (Jan. 1, 2007, to Mar. 13, 2017; and Nov. 1, 2012, to Apr. 27, 2017, respectively). We considered reviews and studies about absolute risk of sexual transmission of HIV between serodiscordant partners to be eligible for inclusion. We used A Measurement Tool to Assess Systematic Reviews (AMSTAR) for review quality, Quality in
Prognosis Studies (QUIPS) instrument for study risk of bias and then the Grading of Recommendations, Assessment, Development and Evaluation (GRADE) approach to assess the quality of evidence across studies. We calculated HIV incidence per 100 person-years with 95\% confidence intervals (Cls). We assigned risk categories according to potential for and evidence of HIV transmission.

RESULTS: We identified 12 reviews. We selected 1 review to estimate risk of HIV transmission for condom use without antiretroviral therapy (1.14 transmissions/ 100 person-years, $95 \% \mathrm{Cl}$ 0.56-2.04; low risk). We identified 11 studies with 23 transmissions over 10511 personyears with antiretroviral therapy (0.22 transmissions/100 person-years, 95\% Cl 0.14-0.33; low risk). We found no transmissions with antiretroviral therapy and a viral load of less than 200 copies $/ \mathrm{mL}$ across consecutive measurements 4 to 6 months apart (0.00 transmissions/ 100 person-years, $95 \% \mathrm{Cl} 0.00-0.28$; negligible risk regardless of condom use).

INTERPRETATION: Based on high-quality evidence, there is a negligible risk of sexual transmission of HIV when an HIVpositive sex partner adheres to antiretroviral therapy and maintains a suppressed viral load of less than 200 copies/mL measured every 4 to 6 months. Sexual transmissions of HIV have occurred when viral load was more than 200 copies $/ \mathrm{mL}$ with antiretroviral therapy or condoms alone were used, although the risk remains low. These findings will help to support patient and clinician decision-making, affect public health case management and contact tracing, and inform justice system responses to HIV nondisclosure. n Canada, an estimated $82 \%$ of people living with HIV acquired their infection through sexual transmission. ${ }^{1}$ Under current Canadian criminal law, people can be prosecuted for not disclosing their HIV-positive status when they engage in sexual activity that poses a "realistic possibility" of HIV transmission. ${ }^{2-5}$

Previous reviews have shown that both antiretroviral therapy and condoms reduce the possibility of HIV transmission. ${ }^{6-8}$ Antiretroviral therapy inhibits viral replication with the goal of suppressing viral load to an undetectable level, defined as a plasma
HIV-1 RNA level below 20-50 copies/mL.9.10 In 2016, an estimated $86 \%$ of the 63110 people living with HIV in Canada were aware of their infection, $81 \%$ of those diagnosed were taking antiretroviral therapy and $91 \%$ of those being treated had a suppressed viral load of less than 200 copies/mL. ${ }^{11}$ Although previous reviews have shown the preventive benefit of antiretroviral therapy, use of condoms or both, they have not included the most recent studies that have changed clinical guidance ${ }^{12-14}$ and public messaging, ${ }^{15}$ and could affect Canadian criminal law. 
To support the examination of the criminal justice system's response to HIV nondisclosure by the Department of Justice Canada, ${ }^{16}$ we synthesized evidence on the absolute risk of HIV transmission during sex between serodiscordant partners. Our objectives were to determine risk when a sex partner who is HIV-positive (Q1) is taking antiretroviral therapy (with varying levels of viral load); (Q2) is taking antiretroviral therapy and has a suppressed viral load; (Q3) is taking antiretroviral therapy and either partner uses condoms (or other barrier methods); (Q4) is taking antiretroviral therapy and has a suppressed viral load, and either partner uses condoms (or other barrier methods); and when (Q5) either partner uses condoms (or barrier methods) alone.

The Public Health Agency of Canada, in consultation with the Department of Justice Canada, chose these questions and the outcome of interest (absolute risk of HIV transmission) as most relevant to informing public health and legal responses.

\section{Methods}

We conducted a systematic review of systematic reviews (overview) and a systematic review of more recent studies that were not available for consideration in previously published reviews (update). Figure 1 presents the analytic framework for the overview and update. We prepared unregistered protocols a priori (Appendices 1 and 2, available at www.cmaj.ca/lookup/suppl/ doi:10.1503/cmaj.180311/-/DC1; summarized in the following section) by following the criteria from the Preferred Reporting Items for Systematic review and Meta-Analysis Protocols
(PRISMA-P). ${ }^{17}$ We followed PRISMA and Cochrane guidance (Supplementary Table S1, Appendix 3, available at www.cmaj.ca/ lookup/suppl/doi:10.1503/cmaj.180311/-/DC1). ${ }^{18,19}$

\section{Overview review}

We developed a comprehensive search strategy (Appendix 1) with assistance from research librarians at Health Canada. We searched MEDLINE, Embase, Global Health and the Cochrane Library from Jan. 1, 2007, to Mar. 13, 2017, in addition to review registries (PROSPERO and Cochrane Library) and reference lists.

We included reviews that provided quantitative estimates of absolute risk of sexual transmission of HIV between serodiscordant sex partners (number of HIV transmissions in a given number of sex acts or person-years). Appendix 1 gives the detailed eligibility criteria.

We performed title and abstract screening, full-text screening, data extraction and quality assessments using the A Measurement Tool to Assess Systematic Reviews (AMSTAR) ${ }^{20}$ independently and in duplicate, with disagreements resolved by a third reviewer. Our AMSTAR ratings informed the selection of reviews for the update.

\section{Update review}

We identified 2 high-quality reviews that addressed Q1 to Q4 to update $^{6,21}$ (see Appendix 2, section 3.3). Research librarians at Health Canada helped to develop the search strategy for the update that encompassed any sexual orientation or type of partnership. The strategy was externally peer reviewed using Peer Review of Electronic Search Strategies (PRESS). ${ }^{22}$ We searched MEDLINE, Embase, the Cochrane Register of Controlled Trials (CENTRAL) and Web of Science from Nov. 1, 2012, to Apr. 27,

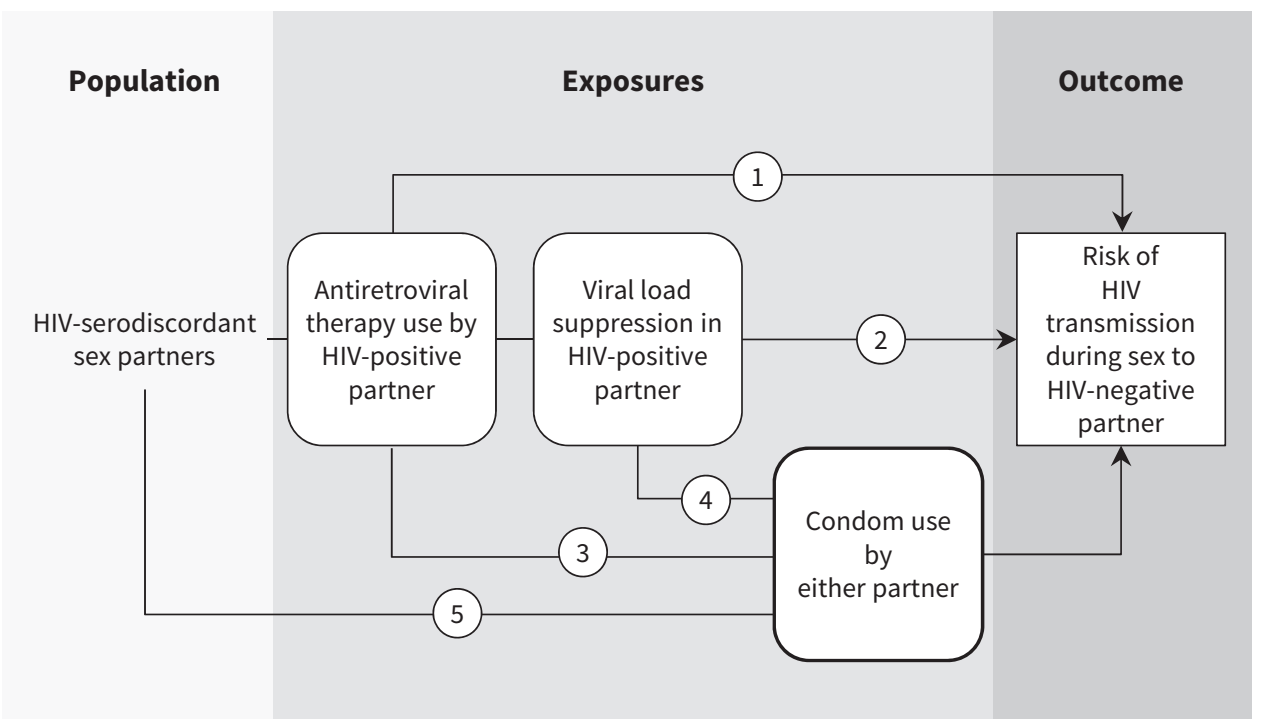

Figure 1: Analytic framework illustrating exposure to preventive measures that reduce the risk of transmission between HIV-serodiscordant sex partners. The numbers in the corresponding pathways illustrate the risk of HIV transmission from the HIV-positive partner to the HIV-negative partner during sex for Q1 to Q5. Note: Q1 = to determine risk when a sex partner who is HIV-positive is taking antiretroviral therapy (with varying levels of viral load); Q2 = to determine risk when a sex partner who is HIV-positive is taking antiretroviral therapy and has a suppressed viral load; Q3 = to determine risk when a sex partner who is HIV-positive is taking antiretroviral therapy and either partner uses condoms (or other barrier methods); Q4 = to determine risk when a sex partner who is HIV-positive is taking antiretroviral therapy and has a suppressed viral load, and either partner uses condoms (or other barrier methods); Q5 = to determine risk when either partner uses condoms (or barrier methods) alone. 
2017, in addition to trial registries and reference lists. We contacted experts to identify unpublished studies. We did not conduct an update for Q5 because a Cochrane review addressing condom use alone ${ }^{7}$ was declared in 2012 to be stable and conclusive evidence not requiring further updates. , $23,24^{2}$

We included studies that reported or provided data to calculate an absolute risk of sexual transmission of HIV between serodiscordant sex partners. These studies included people living with HIV who were taking antiretroviral therapy with monitoring of their viral load and reported on condom use. We combined the primary studies from the update search with those from the reviews. ${ }^{6,21}$ Appendix 2 gives the detailed eligibility criteria.

We performed title and abstract screening, full-text screening, data extraction and study-level risk of bias assessment using the Quality in Prognosis Studies (QUIPS) too ${ }^{25}$ independently and in duplicate, with disagreements resolved by a third reviewer. Our QUIPS ratings informed the risk of bias domain of our overall quality assessments.

We used the Grading of Recommendations, Assessment, Development and Evaluation (GRADE) approach for prognostic studies to assess certainty (i.e., confidence or quality) in the evidence as high, moderate, low or very low. ${ }^{26-28}$ For questions with no direct evidence, we used indirect evidence from other questions, populations and sex acts to estimate transmission risk and rated down for indirectness.

\section{Statistical analysis}

We present descriptive statistics at the study level including aggregate person-years and transmission events. We also describe individual transmission events from all studies in the update. We confirmed that the studies contained independent data. We converted per person-year risk of HIV transmission to incidence per 100 personyears and per-act risk of HIV transmission to incidence per 1000 acts. We calculated incidence of HIV transmission per 100 person-years and exact 95\% confidence intervals (Cls) assuming a Poisson distribution. ${ }^{29}$ We used SAS for all analyses (SAS Enterprise Guide version 5.1, SAS Institute Inc.). When more than 1 cohort was available, we used the sum of transmission events divided by the sum of personyears to calculate a pooled absolute transmission risk (i.e., incidence) and $95 \% \mathrm{Cls}$. We assessed statistical heterogeneity visually with forest plots (because we did not examine comparisons between groups) ${ }^{30}$ to inform the "inconsistency" criterion of our GRADE assessment.

\section{Categories of transmission risk}

To facilitate knowledge translation, we assigned risk categories (no risk, negligible risk, low risk and high risk) to our findings using the criteria outlined in the guideline from the Canadian AIDS Society (Supplementary Table S3, Appendix 3). ${ }^{31}$ We considered 2 criteria when assessing the level of transmission risk associated with an activity: whether there was potential for HIV transmission, and whether there was documented evidence of transmission. ${ }^{31}$

\section{Ethics approval}

Because we systematically reviewed published studies, no ethics approval was required.

\section{Results}

In the overview, of 1414 citations screened, we found 12 systematic reviews ${ }^{6-8,21,32-39}$ that met the eligibility criteria (Supplementary Figure S1, Appendix 3). Table 1 and Supplementary Tables S4-S6 (Appendix 3) summarize characteristics of the included reviews and their key findings (Appendix 4, available at www.cmaj.ca/lookup/suppl/doi:10.1503/cmaj.180311/-/DC1 presents full narrative summaries). Our AMSTAR scores ranged from 2 to 9 out of 11 (Supplementary Table S7, Appendix 3).

We updated 2 reviews on use of antiretroviral therapy ${ }^{6,21}$ that included the same 6 studies; ${ }^{40,44-48} 2$ of those studies had more recent results identified through our update search. ${ }^{49,50}$ of 7266 citations screened in our update, 7 met our eligibility criteria. ${ }^{49-55}$ Therefore, 11 studies (4 from the original reviews ${ }^{4-47}$ and 7 from the update search ${ }^{49-55}$ ) met the eligibility criteria (Supplementary Figure S2, Appendix 3). We identified 13 companion articles (Supplementary Table S20, Appendix 3). ${ }^{40,48,56-66}$

Table 2 (abridged; complete table is available as Supplemenmentary Table S8 in Appendix 3) and Supplementary Tables S9S12 (Appendix 3) summarize study characteristics and key findings (Appendix 5, available at www.cmaj.ca/lookup/suppl/ doi:10.1503/cmaj.180311/-/DC1, presents full narrative summaries). Most of the studies enrolled participants who were 18 years of age or older and had a low or moderate risk of bias (Supplementary Table S13, Appendix 3).

We use the terms "male/man," "female/woman" and "heterosexual" according to the language used by the authors as sex, gender and sexual orientation were not otherwise described. We refer to male serodiscordant couples as "gay, bisexual and other men who have sex with men (MSM)" and to female serodiscordant partners as "lesbian, bisexual and other women who have sex with women (WSW)." No reviews or studies reported on barrier methods other than male condoms, specific sex acts other than penile-vaginal or penile-anal, or HIV transmission risk for WSW. We applied indirect evidence from heterosexual sex partners to WSW for Q1 and Q2, which did not involve male condom use.

Table 3 lists the risk of HIV transmission for Q1 to Q5. Supplementary Figures S3-S5 (Appendix 3) provide forest plots, and Supplementary Tables S14-S19 (Appendix 3) show the GRADE assessments.

\section{Q1: The HIV-positive sex partner is taking antiretroviral} therapy (with varying levels of viral load)

The included studies reported 23 phylogenetically linked HIV transmissions across 10511 person-years of follow-up on antiretroviral therapy (pooled incidence 0.22 transmissions/100 person-years, 95\% Cl 0.14-0.33). ${ }^{44-47,49-55}$ Among heterosexual sex partners, there were 23 linked transmissions over 9922 person-years (pooled incidence 0.23 transmissions/100 person-years, $95 \% \mathrm{Cl} 0.15-0.35) .{ }^{44-}$ $47,49,50,52-55$ We rated the quality of the evidence as high for heterosexual sex partners and as moderate for WSW because of the application of indirect evidence from heterosexual sex partners. Two studies involving MSM found 0 transmissions over 588.96 personyears (pooled incidence 0.00 transmissions/100 person-years, $95 \% \mathrm{Cl} 0.00-0.63) .51,55$ We rated the quality of evidence for MSM as 


\begin{tabular}{|c|c|c|c|c|c|c|c|}
\hline Review, yr & Search performed & Population & $\begin{array}{c}\text { Included study } \\
\text { settings } \\
\text { (by income level)* }\end{array}$ & $\begin{array}{l}\text { Study designs } \\
\text { included }\end{array}$ & $\begin{array}{l}\text { Analysis of } \\
\text { interest }\end{array}$ & $\begin{array}{l}\text { AMSTAR } \\
\text { (score } \\
\text { out of 11) }\end{array}$ & $\begin{array}{c}\text { Other } \\
\text { characteristics }\end{array}$ \\
\hline $\begin{array}{l}\text { Supervie et } \\
\text { al., } 2014^{21}\end{array}$ & $\begin{array}{l}\text { PubMed/MEDLINE, Embase, } \\
\text { Cochrane Library and Web of } \\
\text { Science (up to Aug. 2, 2013) } \\
\text { - IAS conference (2001-2013), } \\
\text { CROI (1993-2013) and IAC } \\
\text { (1985-2012) } \\
\text { - Reference lists of included } \\
\text { articles }\end{array}$ & $\begin{array}{l}\text { Serodiscordant } \\
\text { heterosexual } \\
\text { couples }\end{array}$ & $\begin{array}{l}\text { Primarily } \\
\text { lower-middle- and } \\
\text { upper-middle- } \\
\text { income countries } \\
\text { ( } 2 \text { studies with sites } \\
\text { in high-income } \\
\text { countries) }\end{array}$ & $\begin{array}{l}\text { RCT, } \\
\text { retrospective } \\
\text { and prospective } \\
\text { cohorts }\end{array}$ & $\begin{array}{l}\text { Bayesian } \\
\text { modelling to } \\
\text { develop per-act } \\
\text { risk of HIV } \\
\text { transmission } \\
\text { when the } \\
\text { partner with HIV } \\
\text { is on ART for } \\
\text { more than } 6 \text { mo. }\end{array}$ & 7 & $\begin{array}{l}\text { Studies had to } \\
\text { include } \\
\text { information on } \\
\text { viral load of } \\
\text { partner on ART, } \\
\text { condom use and } \\
\text { sexual activity. }\end{array}$ \\
\hline $\begin{array}{l}\text { Patel et al., } \\
2014^{8}\end{array}$ & $\begin{array}{l}\text { Primary literature search } \\
\text { via MEDLINE, Embase, } \\
\text { CINAHL, Web of Science, } \\
\text { Global Health and Cochrane } \\
\text { Library (January } 2008 \text { to } \\
\text { February 2012) } \\
\text { - Effect modifier search via } \\
\text { PubMed (January } 2008 \text { to } \\
\text { May 2013) } \\
\text { - } 2005 \text { CDC summary, } 2011 \\
\text { British PrEP Guidelines }\end{array}$ & $\begin{array}{l}\text { Serodiscordant } \\
\text { heterosexual } \\
\text { and MSM } \\
\text { couples }\end{array}$ & $\begin{array}{l}\text { Low-, lower- } \\
\text { middle-, } \\
\text { upper-middle- and } \\
\text { high-income } \\
\text { countries }\end{array}$ & $\begin{array}{l}\text { Systematic } \\
\text { review and } \\
\text { meta-analysis, } \\
\text { prospective } \\
\text { cohort, } \\
\text { cross-sectional }\end{array}$ & $\begin{array}{l}\text { Transmission } \\
\text { risk with ART } \\
\text { and/or condoms } \\
\text { calculated by } \\
\text { multiplying } \\
\text { unprotected risk } \\
\text { estimates by } \\
\text { relative risk } \\
\text { reductions of } \\
96 \% \text { (ART), } 80 \% \\
\text { (condoms) or } \\
\text { 99.2\% (ART and } \\
\text { condoms). }\end{array}$ & 2 & $\begin{array}{l}\text { Estimates for } \\
\text { transmission risk } \\
\text { with ART and } \\
\text { condoms derived } \\
\text { from Cohen et al. } \\
\text { (assumed } 96 \% \text { risk } \\
\text { reduction), }{ }^{40} \text { and } \\
\text { Weller and Davis } \\
\text { (80\% risk } \\
\text { reduction), }{ }^{7} \\
\text { respectively. }\end{array}$ \\
\hline $\begin{array}{l}\text { Loutfy et al., } \\
2013^{6}\end{array}$ & $\begin{array}{l}\text { MEDLINE (January } 1950 \text { to } \\
\text { November 2012), Embase } \\
\text { (January } 1980 \text { to November } \\
\text { 2012), CINAHL (January } \\
1980 \text { to November 2012) } \\
\text { and Web of Science } \\
\text { (January } 2004 \text { to November } \\
\text { 2012) } \\
\text { CROI (2008-2011), IAC } \\
\text { (2008-2010), IAS conference } \\
\text { (2009-2011) } \\
\text { - Unspecified journals (June } \\
2010 \text { to November 2012) }\end{array}$ & $\begin{array}{l}\text { Serodiscordant } \\
\text { heterosexual } \\
\text { couples }\end{array}$ & $\begin{array}{l}\text { Primarily } \\
\text { lower-middle- and } \\
\text { upper-middle- } \\
\text { income countries } \\
\text { ( } 2 \text { studies with sites } \\
\text { in high-income } \\
\text { countries) }\end{array}$ & $\begin{array}{l}\text { For } \\
\text { undetectable } \\
\text { VL: } \\
\text { retrospective } \\
\text { and } \\
\text { prospective } \\
\text { cohorts, } \\
\text { cross-sectional } \\
\text { For } \\
\text { unconfirmed } \\
\text { VL: RCT, } \\
2 \text { prospective } \\
\text { cohorts }\end{array}$ & $\begin{array}{l}\text { Fixed-effects } \\
\text { Poisson } \\
\text { regression } \\
\text { model to } \\
\text { develop } \\
\text { summary } \\
\text { statistics for the } \\
\text { effect of ART use } \\
\text { stratified by } \\
\text { confirmed } \\
\text { undetectable VL } \\
\text { and } \\
\text { unconfirmed } \\
\text { undetectable VL. }\end{array}$ & 7 & $\begin{array}{l}\text { Identified studies } \\
\text { did not provide } \\
\text { enough data on } \\
\text { same-sex couples } \\
\text { to generate risk } \\
\text { estimates. } \\
\text { Insufficient } \\
\text { evidence to } \\
\text { develop estimates } \\
\text { for exclusively } \\
\text { condomless sex } \\
\text { (condom use high } \\
\text { in most studies) or } \\
\text { for various sexual } \\
\text { acts. }\end{array}$ \\
\hline $\begin{array}{l}\text { Anglemyer } \\
\text { et al., } 2013^{38}\end{array}$ & $\begin{array}{l}\text { - PubMed, Embase, Cochrane } \\
\text { Central Register of } \\
\text { Controlled Trials, Web of } \\
\text { Science and LILACS (January } \\
1987 \text { to August 2012) } \\
\text { - British HIV/AIDS Association } \\
\text { conference (2001-2008), } \\
\text { CROI (1994-2008), } \\
\text { European AIDS Society } \\
\text { conference (2001, 2003), IAS } \\
\text { conference (2001-2005), IAC } \\
\text { (1985-2004), US National } \\
\text { HIV Prevention Conference } \\
\text { (1999, 2003, 2005) } \\
\text { - Reference lists of included } \\
\text { studies }\end{array}$ & $\begin{array}{l}\text { Serodiscordant } \\
\text { couples (most } \\
\text { were } \\
\text { heterosexual) }\end{array}$ & $\begin{array}{l}\text { Primarily } \\
\text { lower-middle- and } \\
\text { upper-middle- } \\
\text { income countries } \\
\text { ( } 3 \text { studies with sites } \\
\text { in high-income } \\
\text { countries) }\end{array}$ & $\begin{array}{l}\mathrm{RCT} \text {, } \\
\text { prospective } \\
\text { and } \\
\text { retrospective } \\
\text { cohorts }\end{array}$ & $\begin{array}{l}\text { Summary rate } \\
\text { ratios across } \\
\text { studies used to } \\
\text { calculate } \\
\text { absolute } \\
\text { incidence rates } \\
\text { for ART use } \\
\text { based on } \\
\text { baseline } \\
\text { incidence in } \\
\text { control group. }\end{array}$ & 9 & $\begin{array}{l}\text { Risks stratified } \\
\text { based on linked } \\
\text { and unlinked HIV } \\
\text { transmissions, and } \\
\text { by CD4 cell count } \\
\text { subgroups. } \\
\text { Unable to estimate } \\
\text { levels of ART use } \\
\text { (i.e., adherence) or } \\
\text { prevalence of } \\
\text { condom use. }\end{array}$ \\
\hline $\begin{array}{l}\text { Baggaley et } \\
\text { al., } 2013^{36}\end{array}$ & $\begin{array}{l}\text { - PubMed, Science Direct and } \\
\text { NLM Gateway (up to July } \\
\text { 2011) } \\
\text { - IAS and ISSTR conferences, } \\
\text { and CROI (2010, 2011) } \\
\text { - Bibliographies of included } \\
\text { studies }\end{array}$ & $\begin{array}{l}\text { Serodiscordant } \\
\text { heterosexual } \\
\text { couples }\end{array}$ & $\begin{array}{l}\text { Primarily low- and } \\
\text { lower-middle- } \\
\text { income countries } \\
\text { (1 study with site in } \\
\text { high-income } \\
\text { country) }\end{array}$ & $\begin{array}{l}\text { For studies } \\
\text { including a no } \\
\text { ART arm: RCT, } \\
\text { prospective } \\
\text { cohort } \\
\text { For studies } \\
\text { with no } \\
\text { comparison } \\
\text { group with } \\
\text { respect to ART } \\
\text { use: } \\
\text { prospective } \\
\text { cohort }\end{array}$ & $\begin{array}{l}\text { Random-effects } \\
\text { Poisson } \\
\text { regression } \\
\text { model for } \\
\text { summary } \\
\text { statistics of risk } \\
\text { with ART use, } \\
\text { stratified by } \\
\text { setting (high v. } \\
\text { low-middle } \\
\text { income). } \\
\text { No summary } \\
\text { statistics } \\
\text { calculated for } \\
\text { studies with no } \\
\text { comparison } \\
\text { group. }\end{array}$ & 3 & $\begin{array}{l}\text { Separate analyses } \\
\text { for studies with a } \\
\text { comparator (no } \\
\text { ART) group and for } \\
\text { those with no } \\
\text { comparison group. } \\
\text { Studies with no } \\
\text { comparison group } \\
\text { had a variety of } \\
\text { reported ART use } \\
\text { levels. }\end{array}$ \\
\hline
\end{tabular}


Table 1 (part 2 of 2): Characteristics of included reviews

\begin{tabular}{|c|c|c|c|c|c|c|c|}
\hline Review, yr & Search performed & Population & $\begin{array}{c}\text { Included study } \\
\text { settings } \\
\text { (by income level)* }\end{array}$ & $\begin{array}{l}\text { Study designs } \\
\text { included }\end{array}$ & $\begin{array}{c}\text { Analysis of } \\
\text { interest }\end{array}$ & $\begin{array}{l}\text { AMSTAR } \\
\text { (score } \\
\text { out of 11) }\end{array}$ & $\begin{array}{c}\text { Other } \\
\text { characteristics }\end{array}$ \\
\hline $\begin{array}{l}\text { Baggaley et } \\
\text { al., } 2010^{37}\end{array}$ & $\begin{array}{l}\text { - PubMed, Science Direct and } \\
\text { NLM Gateway (up to } \\
\text { September 2008) as } \\
\text { reported in Boily et al. } \text {. }^{35} \\
\text { - Bibliographies of included } \\
\text { studies }\end{array}$ & $\begin{array}{l}\text { Serodiscordant } \\
\text { heterosexual } \\
\text { couples and } \\
\text { MSM, and } \\
\text { individuals at } \\
\text { risk of HIV }\end{array}$ & $\begin{array}{l}\text { Low-, lower- } \\
\text { middle-, } \\
\text { upper-middle-and } \\
\text { high-income } \\
\text { countries } \\
\text { Low-income } \\
\text { countries were used } \\
\text { to derive the ART } \\
\text { risk estimate. }\end{array}$ & $\begin{array}{l}\text { Prospective } \\
\text { and } \\
\text { retrospective } \\
\text { cohorts }\end{array}$ & $\begin{array}{l}\text { Two models } \\
\text { used to } \\
\text { estimate risk } \\
\text { with successful } \\
\text { ART (resulting in } \\
\text { viral } \\
\text { suppression) }\end{array}$ & 3 & $\begin{array}{l}\text { Estimates for ART } \\
\text { based on } 2 \text { models } \\
\text { derived from single } \\
\text { studies of } \\
\text { heterosexual } \\
\text { transmission in } \\
\text { Uganda and } \\
\text { Zambia. }{ }^{41,42}\end{array}$ \\
\hline $\begin{array}{l}\text { Attia et al., } \\
2009^{39}\end{array}$ & $\begin{array}{l}\text { - MEDLINE, Embase (January } \\
1996 \text { to February 2009) } \\
\text { - IAS conference (2001-2008), } \\
\text { CROI (1997-2009) }\end{array}$ & $\begin{array}{l}\text { Serodiscordant } \\
\text { heterosexual } \\
\text { couples }\end{array}$ & $\begin{array}{l}\text { Primarily low- and } \\
\text { upper-middle- } \\
\text { income ( } 3 \text { studies } \\
\text { with sites in } \\
\text { high-income } \\
\text { countries) }\end{array}$ & $\begin{array}{l}\text { Prospective } \\
\text { cohort }\end{array}$ & $\begin{array}{l}\text { Random-effects } \\
\text { Poisson } \\
\text { regression for } \\
\text { effect of ART } \\
\text { and viral load }\end{array}$ & 4 & $\begin{array}{l}\text { Stratified by ART, } \\
\text { no ART and ART at } \\
\text { various VLs. } \\
\text { Authors were } \\
\text { unable to control } \\
\text { for condom use. }\end{array}$ \\
\hline $\begin{array}{l}\text { Boily et al., } \\
2009^{35}\end{array}$ & $\begin{array}{l}\text { - Science Direct and NLM } \\
\text { Gateway (up to September } \\
\text { 2006) } \\
\text { - PubMed (up to September } \\
\text { 2008) } \\
\text { - Bibliographies of relevant } \\
\text { articles }\end{array}$ & $\begin{array}{l}\text { Serodiscordant } \\
\text { heterosexual } \\
\text { couples } \\
\text { including sex } \\
\text { workers and } \\
\text { their clients } \\
\text { and individuals } \\
\text { at risk of HIV }\end{array}$ & $\begin{array}{l}\text { Low-, lower- } \\
\text { middle-, } \\
\text { upper-middle- and } \\
\text { high-income } \\
\text { countries }\end{array}$ & $\begin{array}{l}\text { Prospective } \\
\text { and } \\
\text { retrospective } \\
\text { cohorts, } \\
\text { cross-sectional }\end{array}$ & $\begin{array}{l}\text { Univariate } \\
\text { meta-regression } \\
\text { for condom } \\
\text { effectiveness }\end{array}$ & 5 & $\begin{array}{l}\text { Control versus no } \\
\text { control for condom } \\
\text { use. Condom use } \\
\text { was considered } \\
\text { controlled for if } \\
\text { any attempt was } \\
\text { made to account } \\
\text { for frequent } \\
\text { condom use or if } \\
\text { condom use was } \\
\text { very low. }\end{array}$ \\
\hline $\begin{array}{l}\text { Powers et } \\
\text { al., } 2008^{32}\end{array}$ & $\begin{array}{l}\text { - PubMed/MEDLINE and Web } \\
\text { of Science (up to April 2008) }\end{array}$ & $\begin{array}{l}\text { Serodiscordant } \\
\text { heterosexual } \\
\text { couples and } \\
\text { individuals at } \\
\text { risk of HIV }\end{array}$ & $\begin{array}{l}\text { Low-, lower- } \\
\text { middle-, } \\
\text { upper-middle- and } \\
\text { high-income } \\
\text { countries }\end{array}$ & $\begin{array}{l}\text { Prospective } \\
\text { cohort, } \\
\text { cross-sectional }\end{array}$ & $\begin{array}{l}\text { Univariate } \\
\text { meta-regression } \\
\text { for condom } \\
\text { effectiveness }\end{array}$ & 3 & $\begin{array}{l}\text { Compared "some" } \\
\text { condom use with } \\
\text { "rare" condom use } \\
\text { or adjusted for } \\
\text { condom use }\end{array}$ \\
\hline $\begin{array}{l}\text { Weller and } \\
\text { Davis, } 2002^{7}\end{array}$ & $\begin{array}{l}\text { - AIDSLINE (1980 to June } \\
\text { 2000), CINAHL (1982 to } \\
\text { March 2000), Embase (1974 } \\
\text { to June 2000) and MEDLINE } \\
\text { (1966 to July 2000) } \\
\text { - Review was declared stable } \\
\text { on Jan. 24, 2012, with } \\
\text { evidence described as } \\
\text { "conclusive." Review will no } \\
\text { longer be updated. }\end{array}$ & $\begin{array}{l}\text { Serodiscordant } \\
\text { heterosexual } \\
\text { couples }\end{array}$ & $\begin{array}{l}\text { Low-, lower-middle- } \\
\text { and high-income } \\
\text { countries }\end{array}$ & $\begin{array}{l}\text { Prospective } \\
\text { and } \\
\text { retrospective } \\
\text { cohorts, case } \\
\text { study }\end{array}$ & $\begin{array}{l}\text { Summary } \\
\text { statistic for } \\
\text { condom } \\
\text { effectiveness }\end{array}$ & 4 & $\begin{array}{l}\text { Compared "never" } \\
\text { condom use to } \\
\text { "always" condom } \\
\text { use as reported in } \\
\text { the included } \\
\text { studies }\end{array}$ \\
\hline $\begin{array}{l}\text { Liu et al., } \\
2014^{33}\end{array}$ & $\begin{array}{l}\text { PubMed, Wanfang Data, } \\
\text { China National Knowledge } \\
\text { Infrastructure and Chinese } \\
\text { Biomedical Literature } \\
\text { Database (up to March } \\
\text { 2014) }\end{array}$ & $\begin{array}{l}\text { Serodiscordant } \\
\text { heterosexual } \\
\text { couples }\end{array}$ & $\begin{array}{l}\text { Upper-middle- } \\
\text { income country }\end{array}$ & $\begin{array}{l}\text { Prospective } \\
\text { cohort }\end{array}$ & $\begin{array}{l}\text { Random-effects } \\
\text { Poisson } \\
\text { regression } \\
\text { models to } \\
\text { produce pooled } \\
\text { estimates of HIV } \\
\text { incidence on } \\
\text { ART and with } \\
\text { condom use. }\end{array}$ & 3 & $\begin{array}{l}\text { On ART compared } \\
\text { with no ART and } \\
\text { "consistent" } \\
\text { condom use } \\
\text { compared with } \\
\text { inconsistent } \\
\text { condom use (based } \\
\text { on self-report) }\end{array}$ \\
\hline $\begin{array}{l}\text { Cambiano et } \\
\text { al., } 2013^{34}\end{array}$ & $\begin{array}{l}\text { Web of Science, MEDLINE, } \\
\text { BIOSIS Citation Index, } \\
\text { BIOSIS Previews and } \\
\text { Journal Citation Report } \\
\text { (2006 and November 2013) } \\
\text { Bibliographies of included } \\
\text { articles and studies known } \\
\text { to authors }\end{array}$ & $\begin{array}{l}\text { Serodiscordant } \\
\text { heterosexual } \\
\text { couples }\end{array}$ & $\begin{array}{l}\text { Low-, lower- } \\
\text { middle-, } \\
\text { upper-middle- and } \\
\text { high-income } \\
\text { countries }\end{array}$ & $\begin{array}{l}\text { Systematic } \\
\text { review and } \\
\text { meta-analysis, } \\
\text { RCT, } \\
\text { prospective } \\
\text { and } \\
\text { retrospective } \\
\text { cohorts, } \\
\text { cross- } \\
\text { sectional, } \\
\text { mathematical } \\
\text { model }\end{array}$ & $\begin{array}{l}\text { Narrative } \\
\text { synthesis of } \\
\text { various HIV } \\
\text { transmission } \\
\text { risk and } \\
\text { incidence } \\
\text { estimates for } \\
\text { ART use for } \\
\text { various acts }\end{array}$ & 2 & $\begin{array}{l}\text { No direct evidence } \\
\text { for HIV } \\
\text { transmission risk } \\
\text { for MSM was } \\
\text { available. Cites } \\
\text { other reviews } \\
\text { identified in this } \\
\text { overview. } 6,36,38,39\end{array}$ \\
\hline
\end{tabular}

Note: AMSTAR = A Measurement Tool to Assess Systematic Reviews, ART = antiretroviral therapy, CDC = US Centers for Disease Control and Prevention, CINAHL = Cumulative Index to Nursing and Allied Health Literature, CROI = Conference on Retroviruses and Opportunistic Infections, IAC = International AIDS Conference, IAS = International AIDS Society, ISSTR = International Society of Sexually Transmitted Research, LILACS = Latin American and Caribbean Health Sciences Literature database, MSM = men who have sex with men, NLM = National Library of Medicine, PrEP = Pre-Exposure Prophylaxis, RCT = randomized controlled trial, VL = viral load.

*Study setting groupings by income level based on the World Bank's country and lending group classification for the 2017 fiscal year. ${ }^{43}$ 


\section{Table 2 (part 1 of 2): Characteristics of included studies}

\begin{tabular}{|c|c|c|c|c|c|c|c|c|c|c|}
\hline $\begin{array}{l}\text { Study, yr; } \\
\text { type of } \\
\text { couple }\end{array}$ & $\begin{array}{c}\text { Study } \\
\text { design and } \\
\text { period }\end{array}$ & $\begin{array}{l}\text { Study } \\
\text { location }\end{array}$ & $\begin{array}{l}\text { No. }(\%) \text { of } \\
\text { HIV-positive } \\
\text { partners, by } \\
\text { sex }\end{array}$ & $\begin{array}{c}\text { Adherence } \\
\text { to ART }\end{array}$ & $\begin{array}{l}\text { Timeline for } \\
\text { viral load } \\
\text { testing }\end{array}$ & $\begin{array}{l}\text { Limit of } \\
\text { detection } \\
\text { for VL } \\
\text { assay, } \\
\text { copies/mL }\end{array}$ & $\begin{array}{l}\text { Condom use } \\
\text { reported by } \\
\text { HIV-positive } \\
\text { partners on } \\
\text { ART, No. }(\%)^{\star}\end{array}$ & $\begin{array}{l}\text { No. }(\%) \text { of } \\
\text { partners } \\
\text { with STIs }\end{array}$ & $\begin{array}{c}\text { Frequency } \\
\text { of testing } \\
\text { for HIV }\end{array}$ & $\begin{array}{c}\text { Primary } \\
\text { outcome(s) } \\
\text { of interest }\end{array}$ \\
\hline \multicolumn{11}{|c|}{ Randomized controlled trial } \\
\hline $\begin{array}{l}\text { Cohen et al., } \\
2016^{50} \text { (HIV } \\
\text { Prevention } \\
\text { Trial } \\
\text { Network } \\
\text { [HPTN] 052); } \\
\text { Het }(97 \%)\end{array}$ & $\begin{array}{l}\text { RCT: } \\
2005-2015 \dagger\end{array}$ & $\begin{array}{l}\text { Botswana, } \\
\text { Brazil, India, } \\
\text { Kenya, } \\
\text { Malawi, } \\
\text { South } \\
\text { Africa, } \\
\text { Thailand, } \\
\text { US, } \\
\text { Zimbabwe }\end{array}$ & $\begin{array}{l}\text { At baseline: } \\
\text { F: } 873(49.5) \\
\text { M: } 890(50.5)\end{array}$ & $\begin{array}{l}\text { At interim } \\
\text { analysis ( } 2011) \text {, } \\
79 \% \text { and } 74 \% \\
\text { of early and } \\
\text { delayed arms, } \\
\text { respectively, } \\
\text { reported } 95 \% \\
\text { adherence as } \\
\text { measured by } \\
\text { pill counts. } \ddagger\end{array}$ & $\begin{array}{l}\text { Baseline; every } \\
\text { mo for the first } \\
3 \text { mo; every } \\
3 \text { mo after; at } \\
\text { seroconversion }\end{array}$ & 400 & $\begin{array}{l}100 \% \text { condom } \\
\text { use reported at } \\
\text { interim analysis: } \\
\text { early ART arm, } \\
\text { 96\%; delayed ART } \\
\text { arm, } 95 \%\end{array}$ & $\begin{array}{l}\text { Cumulative } \\
\text { incidence for } \\
\text { syphilis: } \\
\text { HIV+: } 144 \\
\text { (8.2), HIV-: } \\
\text { 103 (5.7); } \\
\text { for gonorrhea: } \\
\text { HIV+: } 102(5.8) \\
\text { HIV-: } 74 \text { (4.1) }\end{array}$ & Every 3 mo & $\begin{array}{l}\text { HIV } \\
\text { incidence on } \\
\text { ART }\end{array}$ \\
\hline \multicolumn{11}{|c|}{ Observational study } \\
\hline $\begin{array}{l}\text { Grulich et } \\
\text { al., } 2015^{51} \\
\text { (Opposites } \\
\text { Attract } \\
\text { Study); MSM }\end{array}$ & $\begin{array}{l}\text { Prospective } \\
\text { cohort: } \\
\text { 2012- } \\
\text { ongoing }\end{array}$ & $\begin{array}{l}\text { Australia, } \\
\text { Thailand, } \\
\text { Brazil }\end{array}$ & $\begin{array}{l}\text { Total enrolled: } \\
\text { M: } 234 \text { (100.0) }\end{array}$ & NR & $\begin{array}{l}\text { Baseline; } \\
\text { follow-up } \\
\text { ( } \geq 2 \text { peryr); at } \\
\text { seroconversion }\end{array}$ & $\begin{array}{l}20-150 \\
\text { based on } \\
\text { the study } \\
\text { site }\end{array}$ & $\begin{array}{l}\text { NR; reports on } \\
\text { condomless acts }\end{array}$ & $\begin{array}{l}\text { Baseline STI } \\
\text { prevalence: } \\
\text { HIV+: } 17 \S \\
(11.2) \\
\text { HIV-: } 10 \S \\
(6.6)\end{array}$ & $\begin{array}{l}\text { Baseline; } \\
\text { follow-up } \\
\text { ( } \geq 2 \text { per yr) }\end{array}$ & $\begin{array}{l}\text { HIV } \\
\text { incidence on } \\
\text { ART by VL } \\
\text { and type of } \\
\text { sex act }\end{array}$ \\
\hline $\begin{array}{l}\text { Rodger et } \\
\text { al., 201655 } \\
\text { (Partners of } \\
\text { People on } \\
\text { ART-A } \\
\text { New } \\
\text { Evaluation } \\
\text { of Risks } \\
\text { [PARTNER] } \\
\text { Study); Het/ } \\
\text { MSM }\end{array}$ & $\begin{array}{l}\text { Prospective } \\
\text { cohort: } \\
\text { Het: } \\
\text { 2010-2014 } \\
\text { MSM: } \\
\text { 2010- } \\
\text { ongoing }\end{array}$ & $\begin{array}{l}\text { Austria, } \\
\text { Belgium, } \\
\text { Denmark, } \\
\text { Finland, } \\
\text { France, } \\
\text { Germany, } \\
\text { Ireland, Italy, } \\
\text { Portugal, } \\
\text { Spain, } \\
\text { Sweden, } \\
\text { Switzerland, } \\
\text { Netherlands, } \\
\text { UK }\end{array}$ & $\begin{array}{l}\text { Het: } \\
\text { F: } 279(31.4 \S) \\
\text { M: } 269(30.3 \S) \\
\text { MSM: } \\
340(38.3 \S)\end{array}$ & $\begin{array}{l}93 \% \text { of Het } \\
\text { men, } 94 \% \text { of } \\
\text { Het women } \\
\text { and } 97 \% \text { of } \\
\text { MSM } \\
\text { self-reported } \\
>90 \% \\
\text { adherence }\end{array}$ & Every 4-6 mo & 50 & $\begin{array}{l}\text { NR; reports on } \\
\text { condomless acts }\end{array}$ & $\begin{array}{l}\text { Any STI at } \\
\text { follow-up: } \\
\text { Het: } \\
\text { HIV+: } 32(5.8) \\
\text { HIV-: } 33(6.0) \\
\text { MSM: } \\
\text { HIV+: } 59 \\
\text { (17.4) } \\
\text { HIV-: } 56 \\
(16.5)\end{array}$ & $\begin{array}{l}\text { Every } \\
4-6 \text { mo }\end{array}$ & $\begin{array}{l}\text { HIV } \\
\text { incidence on } \\
\text { ART and } \\
\text { virally } \\
\text { suppressed } \\
\text { by type of } \\
\text { sex act }\end{array}$ \\
\hline $\begin{array}{l}\text { Mujugira et } \\
\text { al., 2016 } \\
\text { (Partners } \\
\text { Pre-Exposure } \\
\text { Prophylaxis } \\
\text { [PrEP] } \\
\text { Study); Het }\end{array}$ & $\begin{array}{l}\text { Prospective } \\
\text { cohort: } \\
\text { 2008-2012 }\end{array}$ & $\begin{array}{l}\text { Kenya, } \\
\text { Uganda }\end{array}$ & $\begin{array}{l}\text { Among those } \\
\text { who started } \\
\text { ART (83\%): } \\
\text { F: } 1062(58.4) \\
\text { M: } 755(41.6)\end{array}$ & NR & $\begin{array}{l}\text { Baseline; } \\
\text { every } 6 \text { mo; } \\
\text { final visit }\end{array}$ & 40 & $\begin{array}{l}\text { HIV- partner } \\
\text { reported } \\
\text { condomless sex: } \\
\text { at } 10.5 \% \text { of visits } \\
\text { during }<6 \text { mo on } \\
\text { ART; at } 9.1 \% \text { of } \\
\text { visits during } \\
>6 \text { mo on ART }\end{array}$ & NR & Every mo & $\begin{array}{l}\text { HIV } \\
\text { incidence on } \\
\text { ART by ART } \\
\text { duration } \\
(\leq 6 \mathrm{mo} \\
>6 \mathrm{mo})\end{array}$ \\
\hline $\begin{array}{l}\text { Birungi et al., } \\
2015^{52} \\
\text { (Highly Active } \\
\text { Antiretroviral } \\
\text { therapy as } \\
\text { Prevention } \\
\text { [HAARP] } \\
\text { Study); Het }\end{array}$ & $\begin{array}{l}\text { Prospective } \\
\text { cohort: } \\
\text { 2009-2011 }\end{array}$ & Uganda & $\begin{array}{l}\text { F: } 255(43.5) \\
\text { M: } 331(56.5)\end{array}$ & $\begin{array}{l}\text { All } 5 \text { linked } \\
\text { transmissions } \\
\text { reported } \\
\text { "never" missing } \\
\text { ART, and 0 pills } \\
\text { missed in last } \\
\text { week }\end{array}$ & Every 6 mo & 20 & $\begin{array}{l}\text { Condom use at } \\
\text { last sex reported } \\
\text { at baseline: } \\
256 \S(73.6) \S\end{array}$ & $\begin{array}{l}\text { HSV-2 at } \\
\text { enrolment: } \\
\text { HIV+: } 510 \S \\
(87.0) \S \\
\text { HIV-: } 470 \S \\
(80.2) \S\end{array}$ & $\begin{array}{l}\text { Baseline; } \\
\text { every } 3 \text { mo }\end{array}$ & $\begin{array}{l}\text { HIV } \\
\text { incidence on } \\
\text { ART by ART } \\
\text { duration: } \\
\text { ( } \leq 3 \mathrm{mo},>3 \\
\text { mo) }\end{array}$ \\
\hline $\begin{array}{l}\text { He et al., } \\
2013 ;{ }^{53} \text { Het }\end{array}$ & $\begin{array}{l}\text { Prospective } \\
\text { cohort: } \\
\text { 2009-2011 }\end{array}$ & China & $\begin{array}{l}\text { Retained in } \\
\text { cohort: } \\
\text { F: } 167 \S(20.5) \\
\text { M: } 646 \S(79.5)\end{array}$ & NR & $\begin{array}{l}\text { Baseline; } \\
\geq 12 \mathrm{mo}\end{array}$ & 50 & $\begin{array}{l}\text { Condom use over } \\
\text { past } 12 \text { mo } \\
\text { reported at } \\
\text { baseline: } \\
\text { Consistent: } 295 \\
\text { (76.4) } \\
\text { Inconsistent: } 52 \\
\text { (13.5) } \\
\text { No sex: } 39(10.1)\end{array}$ & $\begin{array}{l}\text { At baseline: } \\
\text { Syphilis: } \\
\text { HIV+: } 23(3.2) \\
\text { HIV-: } 9(1.2) \\
\text { HSV-2: } \\
\text { HIV+: } 262 \\
(36.4) \\
\text { HIV-: } 226 \\
(30.0)\end{array}$ & $\begin{array}{l}\text { Baseline; } \\
\geq 12 \text { mo }\end{array}$ & $\begin{array}{l}\text { HIV } \\
\text { incidence on } \\
\text { ART }\end{array}$ \\
\hline $\begin{array}{l}\text { Del Romero } \\
\text { et al., 2015; }{ }^{49} \\
\text { Het }\end{array}$ & $\begin{array}{l}\text { Prospective } \\
\text { cohort: } \\
\text { 1989-2010 }\end{array}$ & Spain & $\begin{array}{l}\text { Participants } \\
\text { with follow-up: } \\
\text { F: } 84 \S(17.9) \\
\text { M: } 385(82.1)\end{array}$ & NR & $\begin{array}{l}\text { Baseline; } \\
\text { every } 6 \text { mo }\end{array}$ & $\begin{array}{l}500 \text { before } \\
1999 ; 50 \\
\text { thereafter }\end{array}$ & $\begin{array}{l}\text { Couples with } \\
\text { condomless acts } \\
\text { at follow-up: } \\
115(58.0)\end{array}$ & $\begin{array}{l}\text { Any STI at } \\
\text { follow-up in } \\
\text { either partner: } \\
\text { Total: } 9(1.9) \S \\
\text { On ART: } 2 \\
\text { (1.0)§ }\end{array}$ & $\begin{array}{l}\text { Baseline; } \\
\text { every } 6 \text { mo }\end{array}$ & $\begin{array}{l}\text { HIV } \\
\text { incidence on } \\
\text { ART by } \\
\text { condom use }\end{array}$ \\
\hline $\begin{array}{l}\text { Reynolds et } \\
\text { al., 201147 } \\
\text { (Rakai } \\
\text { Community } \\
\text { Cohort } \\
\text { Study } \\
\text { [RCCS]); Het }\end{array}$ & $\begin{array}{l}\text { Retrospective } \\
\text { cohort: } \\
\text { 2004-2009 }\end{array}$ & Uganda & $\begin{array}{l}\text { F: } 105(42.0) \S \\
\text { M: } 145(58.0)\end{array}$ & NR & Every 6 mo & 400 & $\begin{array}{l}\text { Condom use } \\
\text { reported by } \\
\text { HIV-partner at } \\
\text { follow-up: } \\
\text { Consistent: } 22 \\
\text { (53.7) } \\
\text { Inconsistent: } 14 \\
\text { (34.2) } \\
\text { Never/not in } \\
12 \text { mo: } 5(12.2)\end{array}$ & $\begin{array}{l}\text { Cumulative } \\
\text { incidence of } \\
\text { self-reported } \\
\text { genital ulcer } \\
\text { disease: } \\
\text { HIV+ on ART: } \\
1(2.4)\end{array}$ & Every 12 mo & $\begin{array}{l}\text { HIV } \\
\text { incidence on } \\
\text { ART }\end{array}$ \\
\hline
\end{tabular}


Table 2 (part 2 of 2): Characteristics of included studies

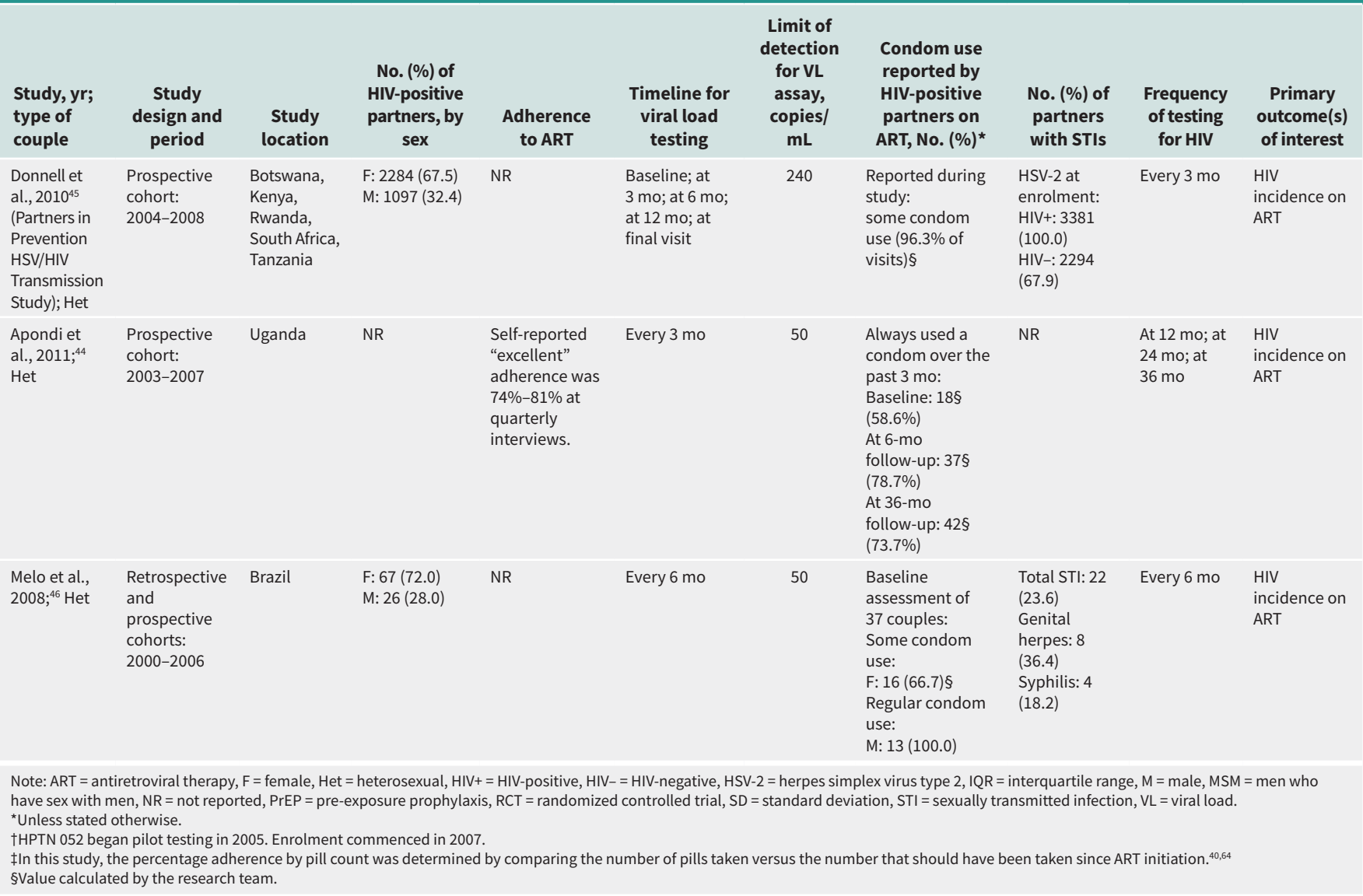

moderate. Based on the criteria from the Canadian AIDS Society, we assigned a low risk of sexual HIV transmission for Q1, because of the potential for transmission during the exchange of bodily fluids, and the small number of reported transmissions in the included studies.

Most of the person-years came from cohorts with high levels of adherence to antiretroviral therapy or viral load suppression (Table 2). Overall condom use varied across studies (Table 2 ) and was not usually characterized for individual participants. Appendix 6 (available at www.cmaj.ca/lookup/suppl/doi:10.1503/cmaj.180311/-/ DC1) presents a narrative description of the 23 linked transmission events. Across all studies (10 511 person-years) where the HIVpositive partner was taking antiretroviral therapy, no linked transmissions were characterized by documented consecutive $(>1)$ viral load measurements of less than 200 copies/mL immediately before or around the estimated time of transmission (Supplementary Table S9, Appendix 3; Appendix 6). Only 2 studies quantified personyears of follow-up with a viral load of less than 200 copies $/ \mathrm{mL}$.

\section{Q2: The HIV-positive sex partner is taking antiretroviral therapy and has a suppressed viral load}

Two studies reported transmission risk when an HIV-positive partner was taking antiretroviral therapy with a suppressed viral load of less than 200 copies/ $\mathrm{mL}$ with measurements taken every 4 to 6 months ${ }^{66}$ or at least twice per year. ${ }^{51}$ During follow-up, studies of sex acts without the use of condoms reported 0 transmissions over 1327 person-years (pooled incidence 0.00 transmissions/100 personyears, $95 \% \mathrm{Cl} 0.00-0.28) .51,55$ Based on high-quality evidence from a single study that included heterosexual sex partners, 0 transmissions occurred over 799 person-years (0.00 transmissions/100 personyears, $95 \% \mathrm{Cl} 0.00-0.46) .{ }^{55}$ We used this estimate as indirect evidence for WSW and rated it as moderate quality. Based on high-quality evidence from both studies that included MSM, we found 0 transmissions over 527.59 person-years (pooled incidence 0.00 transmissions/ 100 person-years, $95 \% \mathrm{Cl} 0.00-0.70) .{ }^{51,55}$ We assigned a negligible risk of sexual HIV transmission for Q2, when an HIV-positive partner is taking antiretroviral therapy and has a suppressed viral load of less than 200 copies $/ \mathrm{mL}$ on consecutive measurements 4 to 6 months apart, because, despite the potential for transmission during the exchange of bodily fluids, there were no reported transmissions in the included studies under these circumstances. We selected a testing interval of 4 to 6 months given that greater than $90 \%$ of the person-years occurred under these conditions.

The same 2 studies ${ }^{51,55}$ reported HIV transmissions per personyears of specific sex acts without condoms, although the followup periods for these acts may not have been mutually exclusive (Supplementary Tables S10, S11 and S14-S19, Appendix 3). In the absence of direct evidence for oral sex, we applied estimates for unspecified heterosexual sex. 


\begin{tabular}{|c|c|c|c|c|}
\hline \multirow[b]{2}{*}{ Question } & Evidence & Absolute risk estimate & \multirow[b]{2}{*}{ Quality of evidence } & \multirow[b]{2}{*}{ Risk assessment } \\
\hline & $\begin{array}{l}\text { No. of HIV } \\
\text { transmissions/ } \\
\text { person-years }\end{array}$ & $\begin{array}{l}\text { No. of HIV transmissions per } \\
100 \text { person-years }(95 \% \mathrm{CI})\end{array}$ & & \\
\hline 1. $\mathrm{ART}^{\star}$ & $\begin{array}{l}\text { Het: } 23 / 9922 \\
\text { MSM: } 0 / 588.96 \\
\text { WSW: no direct evidence } \\
\text { Overall: } 23 / 10511\end{array}$ & $\begin{array}{l}\text { Het/WSW: } 0.23(0.15-0.35) \\
\text { MSM: } 0.00(0.00-0.63) \\
\text { Overall: } 0.22(0.14-0.33)\end{array}$ & $\begin{array}{l}\text { Het: high } \\
\text { MSM: moderate owing to } \\
\text { serious indirectness } \ddagger \text { and } \\
\text { imprecision§ } \\
\text { WSW: moderate owing to } \\
\text { very serious indirectness }\end{array}$ & $\begin{array}{l}\text { Low risk of transmission } \\
\text { (potential for transmission; } \\
\text { few reports of } \\
\text { transmission) }\end{array}$ \\
\hline $\begin{array}{l}\text { 2. ART + viral } \\
\text { suppressiont }\end{array}$ & $\begin{array}{l}\text { Het: } 0 / 799 \\
\text { MSM: } 0 / 527.59 \\
\text { WSW: no direct evidence } \\
\text { Overall: } 0 / 1327\end{array}$ & $\begin{array}{l}\text { Het/WSW: } 0.00(0.00-0.46) \\
\text { MSM: } 0.00(0.00-0.70) \\
\text { Overall: } 0.00(0.00-0.28)\end{array}$ & $\begin{array}{l}\text { Het/MSM: high } \\
\text { WSW: moderate owing to } \\
\text { serious indirectness }{ }^{\star \star} \text { and } \\
\text { imprecision§ }\end{array}$ & $\begin{array}{l}\text { Negligible risk of } \\
\text { transmission (potential for } \\
\text { transmission; no } \\
\text { confirmed transmission) }\end{array}$ \\
\hline 3. $\mathrm{ART}^{\star}+$ condom & Not available & $\begin{array}{l}0.003(0.00-0.03) \text { to } 0.11(0.02-0.73) \\
\text { per } 1000 \text { acts (depending on } \\
\text { population and sex acts in question) }\end{array}$ & $\begin{array}{l}\text { Modelled estimate from } \\
\text { Patel et al., } 2014^{8}\end{array}$ & $\begin{array}{l}\text { Low risk of transmission } \\
\text { for penile-vaginal and } \\
\text { penile-anal sex (potential } \\
\text { for transmission; few } \\
\text { reports of transmission) }\end{array}$ \\
\hline $\begin{array}{l}\text { 4. ART + viral } \\
\text { suppression }++ \\
\text { condom }\end{array}$ & $\begin{array}{l}\text { No direct evidence } \\
\text { Indirect evidence from } \\
\text { Q2 was used. }\end{array}$ & $\begin{array}{l}\text { Het: } 0.00(0.00-0.46) \\
\text { MSM: } 0.00(0.00-0.70) \\
\text { Overall: } 0.00(0.00-0.28)\end{array}$ & $\begin{array}{l}\text { Het/MSM: moderate owing } \\
\text { to serious indirectness } † \dagger \\
\text { and imprecision§ }\end{array}$ & $\begin{array}{l}\text { Negligible risk of } \\
\text { transmission (potential for } \\
\text { transmission; no } \\
\text { confirmed transmission) }\end{array}$ \\
\hline 5. Condom & Het: $11 / 946.3$ & $1.14(0.56-2.04)$ & $\begin{array}{l}\text { Stable and conclusive } \\
\text { evidence from a Cochrane } \\
\text { review }^{7}\end{array}$ & $\begin{array}{l}\text { Low risk of transmission } \\
\text { (potential for transmission; } \\
\text { few reports of } \\
\text { transmission) }\end{array}$ \\
\hline
\end{tabular}

Note: $\mathrm{ART}=$ antiretroviral therapy, $\mathrm{Cl}=$ confidence interval, $\mathrm{Het}=$ heterosexual couples, $\mathrm{MSM}=$ men who have sex with men, $\mathrm{Q} 2=$ to determine risk when a sex partner who is HIV-positive is taking antiretroviral therapy and has a suppressed viral load, WSW = women who have sex with women.

*Viral load levels were variable.

†Viral load $<200$ copies $/ \mathrm{mL}$ as per data in the included studies.

fIndirectness was rated serious because almost all follow-up in both studies occurred under conditions of viral load suppression; therefore, the population does not fully reflect a population of individuals on ART with varying levels of viral load.

§Imprecision was rated serious because we considered the sample size and follow-up time to be insufficient (i.e., $<2000$ participants and $<4000$ person-years).

IIndirectness was rated very serious because the studies did not account consistently for relevant confounding variables (e.g., condom use, type of sex act, frequency of sex act,

sexually transmitted infections, injection drug use, duration on ART and viral load), and the estimates were from a different population (i.e., heterosexual sex partners).

${ }^{\star *}$ Indirectness was rated serious because the estimates were from a different population (i.e., heterosexual sex partners).

$\dagger \dagger$ Indirectness was rated serious because the exposure does not directly match the question as studies contributing to this estimate included couples performing sex acts without condom use.

\section{Q3: The HIV-positive sex partner is taking antiretroviral therapy (with varying levels of viral load) and either partner uses condoms}

No studies reported empirical estimates for Q3; however, a systematic review published in $2014^{8}$ modelled the combined effect of antiretroviral therapy and condoms to derive per-act risks, ranging from 0.003 transmissions per 1000 acts $(95 \% \mathrm{Cl} 0.00-$ 0.03 ) for insertive vaginal sex to 0.11 transmissions per 1000 acts (95\% Cl 0.02-0.73) for receptive anal sex. Furthermore, 4 transmissions were found in 1 study when the HIV-positive partner was taking antiretroviral therapy and reported consistent condom use, ${ }^{52}$ although there was inadequate information to calculate an estimate of transmission risk. We assigned a low risk of sexual HIV transmission for penile-vaginal sex and penile-anal sex for Q3 because of the potential for transmission during the exchange of bodily fluids that may occur during a slip, break or other incorrect use of the condom when viral load is not suppressed and the few reports of transmission under these circumstances. Given the paucity of evidence, we did not estimate transmission risk for oral sex when antiretroviral therapy (with variable viral load) and condoms were used together.

\section{Q4: The HIV-positive sex partner is taking antiretroviral therapy and has a suppressed viral load, and either partner uses condoms}

No reviews or studies provided direct evidence for Q4. We used indirect evidence from studies that reported on antiretroviral therapy, viral load suppression and sex without the use of condoms to provide a risk estimate (pooled incidence 0.00 transmissions/ 100 person-years, $95 \% \mathrm{Cl} 0.00-0.28) .{ }^{51,55}$ We rated the quality of the indirect evidence as moderate for heterosexual sex partners and MSM. We assigned a negligible risk of sexual HIV 
transmission for Q4, when an HIV-positive partner is taking antiretroviral therapy, has a suppressed viral load of less than 200 copies/mL on consecutive measurements 4 to 6 months apart and uses condoms, per the rationale for Q2.

\section{Q5: Either partner uses condoms alone}

Five reviews provided pooled estimates of the risk of HIV transmission $^{7,8,32,33,35}$ when heterosexual sex partners used condoms (Supplementary Tables S4-S6, Appendix 3). Because the findings of a Cochrane systematic review ${ }^{7}$ were less likely to have been confounded by antiretroviral therapy use and were declared as stable conclusive evidence in 2012 by The Cochrane Collaboration, ${ }^{23,24}$ we did not update this review. The authors of the review ${ }^{7}$ found that among serodiscordant couples who reported "always" using condoms, there were 1.14 HIV transmissions per 100 person-years (95\% Cl 0.56-2.04). We assigned a low risk of sexual HIV transmission for Q5 because there is potential for transmission during the exchange of bodily fluids that may occur during a slip, break or other incorrect condom use, and transmissions have occurred among partners reporting consistent condom use.

\section{Interpretation}

We found high-quality evidence showing a negligible risk of sexual HIV transmission when an HIV-positive sex partner had a suppressed viral load of less than 200 copies/mL that was maintained through adherence to antiretroviral therapy and confirmed on consecutive measurements every 4 to 6 months. In the GRADE approach, high-quality evidence means we are very confident that the true transmission risk is not higher than the upper limit of the reported confidence interval. We found that the risk of sexual HIV transmission is low when antiretroviral therapy (with varying levels of viral load), condoms or both were used. Based on our findings, relevant case law and other factors, the Department of Justice Canada concluded that the criminal law should not apply to people living with HIV who maintain a suppressed viral load of less than 200 copies/mL. ${ }^{5}$ The department also concluded that the criminal law should generally not apply to "persons living with HIV who: are on treatment; are not on treatment but use condoms; or, engage only in oral sex (unless other risk factors are present and the person living with HIV is aware of those risks)." 5

The risk of HIV transmission with varying levels of viral load (Q1) may have been underestimated because most of our analyses were based on cohorts with high levels of adherence to antiretroviral therapy or viral load suppression. There may also have been confounding caused by condom use. For Q2 and Q4, we could not statistically rule out a risk of approximately 27 transmissions in 1000 serodiscordant couples who were followed for 10 years (based on the upper limit of the confidence interval for the estimate); ${ }^{68}$ however, a risk this high is unlikely given there were no confirmed transmissions while the viral load was less than 200 copies/mL on consecutive measurements in any study. Where there were 0 transmission events, the upper limits of the 95\% Cls were solely a function of the studies' person-years of follow-up. $46,47,49,51,55$ For Q3, the transmission risk a priori should be lower than with either antiretroviral therapy or condom use alone, although it does not meet the criteria from the Canadian AIDS Society for negligible risk. Some practices within a particular Canadian AIDS Society risk category may pose a lower risk than others (e.g., Q3 v. Q5). ${ }^{69}$ For Q5, risk of HIV transmission with condom use may have been overestimated (i.e., condom effectiveness is underestimated) because consistent use may have been overreported owing to recall or social desirability bias, confounding by indication (e.g., individuals engaged in higher risk activities more likely to use condoms) and the possible inclusion of nonphylogenetically linked cases in estimates. ${ }^{7}$

Our review had a number of strengths including using an equity-based approach in considering all genders, sexual orientations and relationship types in our searches, and developing conclusions for seldom-studied groups including WSW. Our observed transmission rate for $\mathrm{Q} 1$ was consistent with that found in a 2013 systematic review ${ }^{6}$ (0.14 transmissions/100 personyears, 95\% Cl 0.04-0.31; Supplementary Table S4, Appendix 3). Unlike previous reviews ${ }^{6,21}$ that relied on modelling and assumptions about eligible person-years of follow-up to develop estimates for Q2, our review relied on empirical data. We also included a rating of certainty in our risk estimates, ${ }^{28}$ and assigned levels of risk using a priori objective criteria; ${ }^{31}$ both of which may aid the public, as well as the medical and legal communities, in understanding the risks of transmission.

\section{Limitations}

Our study protocols (Appendices 1 and 2), although developed a priori and peer reviewed, were not registered or published. Our ability to conduct certain analyses (e.g., per-act risk estimates, risk with concomitant sexually transmitted infections or for specific sex acts) was limited by both the level of detail and inconsistent measures across the included studies. Had the data allowed calculation of per-act estimates, however, our conclusions would not have changed. Updates of 2 studies that were included in our review were recently released. ${ }^{51,55,70,71}$ If all of the additional follow-up from these studies were eligible for incorporation into our meta-analysis, the upper confidence interval of our estimate for Q2 and Q4 would be lowered (from 0.28 to 0.13 ), but our point estimate and conclusions would not change. A recent expert consensus has, in any case, cautioned against overreliance on theoretical risks in this context. ${ }^{72}$ Finally, the risk of HIV transmission in study populations may not reflect the risks and circumstances in individual relationships.

\section{Conclusion}

Our findings show that there is a negligible risk of sexually transmitting HIV when an HIV-positive sex partner adheres to antiretroviral therapy and maintains a suppressed viral load of less than 200 copies/mL on consecutive measurements every 4 to 6 months. The risk of sexual HIV transmission is low when an HIVpositive sex partner is taking antiretroviral therapy without a suppressed viral load of less than 200 copies/mL, condoms are used or both. These findings will support individual patient and clinician decision-making, and will have implications for public 
health case management and contact tracing. The Department of Justice Canada used these findings to inform their 2017 report on the justice system's response to HIV nondisclosure, ${ }^{5}$ and they may inform the responses of other justice systems.

\section{References}

1. Summary: Estimates of HIV incidence, prevalence and proportion undiagnosed in Canada, 2014. Ottawa: Public Health Agency of Canada; 2015.

2. R. v. Cuerrier 19982 SCR 371 , British Columbia.

3. R. v. Mabior 2012 SCC 47, Manitoba.

4. R. v. DC 2012 SCC 48, Quebec.

5. Criminal justice system's response to non-disclosure of HIV. Ottawa: Department of Justice Canada; 2017 Dec. 1.

6. Loutfy MR, Wu W, Letchumanan M, et al. Systematic review of HIV transmission between heterosexual serodiscordant couples where the HIV-positive partner is fully suppressed on antiretroviral therapy. PLoS One 2013;8:e55747.

7. Weller S, Davis K. Condom effectiveness in reducing heterosexual HIV transmission. Cochrane Database Syst Rev 2002;(1):CD003255.

8. Patel P, Borkowf CB, Brooks JT, et al. Estimating per-act HIV transmission risk: a systematic review. AIDS 2014;28:1509-19.

9. Günthard HF, Saag MS, Benson CA, et al. Antiretroviral drugs for treatment and prevention of HIV infection in adults: 2016 recommendations of the International Antiviral Society-USA Panel. JAMA 2016;316:191-210.

10. Montaner J, Guillemi S, Harris M, editors. Committee for Drug Evaluation and Therapy. Therapeutic guidelines for antiretroviral (ARV) treatment of adult HIV infection. Vancouver: British Columbia Centre for Excellence in HIV/AIDS; September 2015.

11. Summary: Estimates of HIV incidence, prevalence and Canada's progress on meeting the 90-90-90 HIV targets, 2016. Ottawa: Public Health Agency of Canada; 2018.

12. Becker M, Cox J, Evans GA, et al. AMMI Canada position statement: The use of early antiretroviral therapy in HIV-infected persons. Ottawa: Association of Medical Microbiology and Infectious Disease Canada; 2016. Available: www. ammi.ca/Update/39.ENG.pdf (accessed 2017 Mar. 9).

13. Panel on Antiretroviral Guidelines for Adults and Adolescents. Guidelines for the use of antiretroviral agents in HIV-1-infected adults and adolescents. Washington (DC): U.S. Department of Health and Human Services; updated 2018 May 30.

14. Consolidated guidelines on the use of antiretroviral drugs for treating and preventing HIV infection: recommendations for a public health approach. 2nd ed. Geneva: World Health Organization; 2016.

15. Risk of sexual transmission of HIV from a person living with HIV who has an undetectable viral load: messaging primer and consensus statement. Prevention Access Campaign; 2016 July 21 (updated 2018 Aug. 23). Available: www. preventionaccess.org/consensus (accessed 2017 Mar. 9).

16. Minister Wilson-Raybould issues statement on World AIDS Day [statement]. Ottawa: Department of Justice Canada; 2016. Available: www.canada.ca/en/ department-justice/news/2016/12/minister-wilson-raybould-issues-statement -world-aids.html (accessed 2017 Mar. 9).

17. Moher D, Shamseer L, Clarke M, et al.; PRISMA-P Group. Preferred reporting items for systematic review and meta-analysis protocols (PRISMA-P) 2015 statement. Syst Rev 2015;4:1.

18. Moher D, Liberati A, Tetzlaff J, et al.; The PRISMA Group. Preferred reporting items for systematic reviews and meta-analyses: The PRISMA Statement. PLoS Med 2009;6:e1000097.

19. Higgins J, Green S, editors. Cochrane handbook for systematic reviews of interventions. Version 5.1.0. [updated March 2011]. Oxford (UK): The Cochrane Collaboration; 2011.

20. Shea BJ, Grimshaw JM, Wells GA, et al. Development of AMSTAR: a measurement tool to assess the methodological quality of systematic reviews. $B M C$ Med Res Methodol 2007;7:10.

21. Supervie V, Viard JP, Costagliola D, et al. Heterosexual risk of HIV transmission per sexual act under combined antiretroviral therapy: systematic review and bayesian modeling. Clin Infect Dis 2014;59:115-22.

22. McGowan J, Sampson M, Salzwedel DM, et al. PRESS peer review of electronic search strategies: 2015 Guideline Statement. J Clin Epidemiol 2016;75:40-6.

23. What's New events (publishing events) in Cochrane Reviews: new publication, new citation, or change in publication status. Oxford (UK): The Cochrane Collaboration; updated 2018 Aug. 30. Available: http://community.cochrane.org/editorial -and-publishing-policy-resource/cochrane-review-management/what $\%$ E2 $\% 80 \%$ 99s-new-events-publishing-events-cochrane-reviews-new-publication-new-citation -or-change (accessed 2017 Mar. 9).

24. Weller SC, Davis-Beaty K; HIV/AIDS Group. Using condoms consistently reduces sexual transmission of HIV infection. Oxford (UK): The Cochrane Collaboration; 2002. Available: www.ncbi.nlm.nih.gov/pubmedhealth/ PMH0011925/ (accessed 2017 June 2).
25. Hayden JA, van der Windt DA, Cartwright JL, et al. Assessing bias in studies of prognostic factors. Ann Intern Med 2013;158:280-6.

26. Balshem $\mathrm{H}$, Helfand $\mathrm{M}$, Schünemann $\mathrm{HJ}$, et al. GRADE guidelines: 3 . Rating the quality of evidence. J Clin Epidemiol 2011;64:401-6.

27. Schünemann H, Brożek J, Guyatt G, et al., editors. GRADE handbook. GRADE Working Group; 2013. Available: http://gdt.guidelinedevelopment.org/app/ handbook/handbook.html (accessed 2017 June 6).

28. Iorio A, Spencer FA, Falavigna M, et al. Use of GRADE for assessment of evidence about prognosis: rating confidence in estimates of event rates in broad categories of patients. BMJ 2015;350:h870.

29. Ulm K. A simple method to calculate the confidence interval of a standardized mortality ratio (SMR). Am J Epidemiol 1990;131:373-5.

30. Neyeloff JL, Fuchs SC, Moreira LB. Meta-analyses and Forest plots using a microsoft excel spreadsheet: step-by-step guide focusing on descriptive data analysis. BMC Res Notes 2012;5:52.

31. HIV transmission: guidelines for assessing risk - A resource for educators, counsellors and health care providers. 5th ed. Ottawa: Canadian AIDS Society; 2004.

32. Powers KA, Poole C, Pettifor AE, et al. Rethinking the heterosexual infectivity of HIV-1: a systematic review and meta-analysis. Lancet Infect Dis 2008;8:553-63.

33. Liu H, Su Y, Zhu L, et al. Effectiveness of ART and condom use for prevention of sexual HIV transmission in serodiscordant couples: a systematic review and meta-analysis. PLoS One 2014;9:e111175.

34. Cambiano V, O'Connor J, Phillips AN, et al. Antiretroviral therapy for prevention of HIV transmission: implications for Europe. Euro Surveill 2013;18:20647.

35. Boily MC, Baggaley RF, Wang L, et al. Heterosexual risk of HIV-1 infection per sexual act: systematic review and meta-analysis of observational studies. Lancet Infect Dis 2009;9:118-29.

36. Baggaley RF, White RG, Hollingsworth TD, et al. Heterosexual HIV-1 infectiousness and antiretroviral use: Systematic review of prospective studies of discordant couples. Epidemiology 2013;24:110-21.

37. Baggaley RF, White RG, Boily MC. HIV transmission risk through anal intercourse: Systematic review, meta-analysis and implications for HIV prevention. Int J Epidemiol 2010;39:1048-63.

38. Anglemyer A, Rutherford GW, Horvath T, et al. Antiretroviral therapy for prevention of HIV transmission in HIV-discordant couples. Cochrane Database Syst Rev 2013;(4):CD009153.

39. Attia S, Egger M, Müller M, et al. Sexual transmission of HIV according to viral load and antiretroviral therapy: systematic review and meta-analysis. AIDS 2009;23:1397-404.

40. Cohen MS, Chen YQ, McCauley M, et al.; HPTN 052 Study Team. Prevention of HIV-1 infection with early antiretroviral therapy. N Engl J Med 2011;365:493-505.

41. Fideli US, Allen SA, Musonda R, et al. Virologic and immunologic determinants of heterosexual transmission of human immunodeficiency virus type 1 in Africa. AIDS Res Hum Retroviruses 2001;17:901-10.

42. Quinn TC, Wawer MJ, Sewankambo N, et al. Viral load and heterosexual transmission of human immunodeficiency virus type 1. Rakai Project Study Group. N Engl J Med 2000;342:921-9.

43. World Bank country and lending groups. Washington (DC): The World Bank. Available: https://datahelpdesk.worldbank.org/knowledgebase/articles /906519 (accessed 2017 June 2).

44. Apondi R, Bunnell R, Ekwaru JP, et al. Sexual behavior and HIV transmission risk of Ugandan adults taking antiretroviral therapy: 3 year follow-up. AIDS 2011;25:1317-27.

45. Donnell D, Baeten JM, Kiarie J, et al.; Partners in Prevention HSV/HIV Transmission Study Team. Heterosexual HIV-1 transmission after initiation of antiretroviral therapy: a prospective cohort analysis. Lancet 2010;375:2092-8.

46. Melo MG, Santos BR, De Cassia Lira R, et al. Sexual transmission of HIV-1 among serodiscordant couples in Porto Alegre, southern Brazil. Sex Transm Dis 2008;35:912-5

47. Reynolds SJ, Makumbi F, Nakigozi G, et al. HIV-1 transmission among HIV-1 discordant couples before and after the introduction of antiretroviral therapy. AIDS 2011;25:473-7.

48. Del Romero J, Castilla J, Hernando V, et al. Combined antiretroviral treatment and heterosexual transmission of HIV-1: cross sectional and prospective cohort study. BMJ 2010;340:c2205.

49. Del Romero J, Río I, Castilla J, et al. Absence of transmission from HIV-infected individuals with HAART to their heterosexual serodiscordant partners. Enferm Infecc Microbiol Clin 2015;33:666-72.

50. Cohen MS, Chen YQ, McCauley M, et al.; HPTN 052 Study Team. Antiretroviral therapy for the prevention of HIV-1 transmission. N Engl J Med 2016;375:830-9.

51. Grulich AE, Bavington BR, Jin F, et al. HIV transmission in male serodiscordant couples in Australia, Thailand and Brazil. In: Conference on Retroviruses and Opportunistic Infections; 2015 Feb. 23-26; Seattle, Wash.; 2015. Available: www. croiconference.org/sessions/hiv-transmission-in-male-serodiscordant-couples -Australia-Thailand-and-Brazil (accessed 2018 Nov. 5). 
52. Birungi J, Min JE, Muldoon KA, et al. Lack of effectiveness of antiretroviral therapy in preventing HIV infection in serodiscordant couples in Uganda: an observational study. PLoS One 2015;10:e0132182.

53. He N, Duan S, Ding Y, et al. Antiretroviral therapy reduces HIV transmission in discordant couples in rural Yunnan, China. PLoS One 2013;8:e77981.

54. Mujugira A, Celum C, Coombs RW, et al.; Partners PrEP Study Team. HIV transmission risk persists during the first 6 months of antiretroviral therapy. J Acquir Immune Defic Syndr 2016;72:579-84.

55. Rodger AJ, Cambiano V, Bruun T, et al.; PARTNER Study Group. Sexual activity without condoms and risk of HIV transmission in serodifferent couples when the HIV-positive partner is using suppressive antiretroviral therapy. JAMA 2016;316:171-81.

56. Bunnell R, Ekwaru J, King R, et al. 3-year follow-up of sexual behavior and HIV transmission risk of persons taking ART in rural Uganda. In: Proceedings of the Fifteenth Conference on Retroviruses and Opportunistic Infections; 2008 Feb. 3-6; Boston. Abstract 29.

57. Chen YQ, Masse B, Wang L, et al. Statistical considerations for the HPTN 052 Study to evaluate the effectiveness of early versus delayed antiretroviral strategies to prevent the sexual transmission of HIV-1 in serodiscordant couples. Contemp Clin Trials 2012;33:1280-6.

58. Cohen M, Chen Y, McCauley M, et al. Final results of the HPTN 052 randomized controlled trial: antiretroviral therapy prevents HIV transmission. J Int AIDS Soc 2015;18:15.

59. Eshleman SH, Hudelson SE, Redd AD, et al. Treatment as prevention: characterization of partner infections in the HIV Prevention Trials Network 052 trial. J Acquir Immune Defic Syndr 2017;74:112-6

60. Fogel JM, Wang L, Parsons TL, et al. Undisclosed antiretroviral drug use in a multinational clinical trial (HIV Prevention Trials Network 052). J Infect Dis 2013;208:1624-8.

61. Bavinton BR, Jin F, Prestage G, et al.; Opposites Attract Study Group. The Opposites Attract Study of viral load, HIV treatment and HIV transmission in serodiscordant homosexual male couples: design and methods. BMC Public Health 2014;14:917.

62. Baeten JM, Donnell D, Mugo NR, et al.; Partners PrEP Study Team. Single-agent tenofovir versus combination emtricitabine plus tenofovir for pre-exposure prophylaxis for HIV-1 acquisition: an update of data from a randomised, double-blind, phase 3 trial. Lancet Infect Dis 2014;14:1055-64.

63. Ping LH, Jabara CB, Rodrigo AG, et al. HIV-1 transmission during early antiretroviral therapy: evaluation of two HIV-1 transmission events in the HPTN 052 prevention study. PLoS One 2013;8:e71557.

64. Safren SA, Mayer KH, Ou SS, et al.; HPTN 052 Study Team. Adherence to early antiretroviral therapy: results from HPTN 052, a Phase III, multinational randomized trial of ART to prevent HIV-1 sexual transmission in serodiscordant couples. J Acquir Immune Defic Syndr 2015;69:234-40.

65. Mujugira A, Baeten JM, Donnell D, et al.; Partners PrEP Study Team. Characteristics of HIV-1 serodiscordant couples enrolled in a clinical trial of antiretroviral pre-exposure prophylaxis for HIV-1 prevention. PLoS One 2011;6:e25828.

66. Rodger A, Bruun T, Weait M, et al.; PARTNER study group. Partners of people on ART - a New Evaluation of the Risks (The PARTNER study): design and methods. BMC Public Health 2012;12:296.

67. Wawer MJ, Gray RH, Sewankambo NK, et al. Rates of HIV-1 transmission per coital act, by stage of HIV-1 infection, in Rakai, Uganda. J Infect Dis 2005;191: 1403-9.

68. Olsen J, Christensen K, Murrary J, et al. Estimates of associations. In: Ahrens W, Pigeot I, editors. An introduction to epidemiology for health professionals. New York: Springer-Verlag New York; 2010:15-8.

69. Expert consensus: viral load and the risk of HIV transmission [report]. Quebéc: Institut national de santé publique du Québec; 2014.

70. Bavinton BR, Pinto AN, Phanuphak N, et al.; Opposites Attract Study Group. Viral suppression and HIV transmission in serodiscordant male couples: an international, prospective, observational, cohort study. Lancet HIV 2018;5:e438-47.

71. Rodger A, Cambiano V, Bruun T, et al. Risk of HIV transmission through condomless sex in MSM couples with suppressive ART: The PARTNER2 Study extended results in gay men. In: Proceedings of the Twenty-second International AIDS Conference (AIDS 2018); 2018 July 23-27; Amsterdam, the Netherlands. Abstract 13470.

72. Barré-Sinoussi F, Abdool Karim SS, Albert J, et al. Expert consensus statement on the science of HIV in the context of criminal law. J Int AIDS Soc 2018;21: e25161.

\section{Competing interests: None declared.}

This article has been peer reviewed.

Affiliations: Centre for Communicable Diseases and Infection Control (Traversy, Varsaneux, Weekes, Avey, Niragira, Gervais, Rodin), Public Health Agency of Canada; Department of Family Medicine (LeMessurier) and School of Epidemiology and Public Health (LeMessurier), University of Ottawa, Ottawa, Ont.; Department of Health Research Methods, Evidence, and Impact (Guyatt), McMaster University, Hamilton, Ont.

Contributors: Rachel Rodin conceptualized and oversaw this project in response to the Department of Justice Canada's request to the Public Health Agency of Canada for an assessment of the most recent medical science on sexual HIV transmission. Rachel Rodin and Jennifer LeMessurier designed the project with input from Gregory Traversy, Olivia Varsaneux, Makenzie Weekes, Marc Avey and Oscar Niragira. Jennifer LeMessurier, Gregory Traversy, Olivia Varsaneux, Makenzie Weekes and Oscar Niragira screened references and extracted data. Gregory Traversy, Olivia Varsaneux, Makenzie Weekes and Oscar Niragira conducted quality (AMSTAR) and risk-of-bias (QUIPS) assessments. Jennifer LeMessurier, Gregory Traversy, Olivia Varsaneux and Makenzie Weekes assessed the quality of evidence across studies (GRADE) with methodological assistance from Gordon Guyatt. Jennifer LeMessurier, Gregory Traversy, Olivia Varsaneux, Makenzie Weekes, Marc Avey, Robert Gervais and Rachel Rodin analyzed and interpreted data. Jennifer LeMessurier, Gregory Traversy, Olivia Varsaneux, Makenzie Weekes, Marc Avey and Rachel Rodin drafted the manuscript. All of the authors critically revised the manuscript for important intellectual content, gave final approval of the version to be published and agreed to be accountable for all aspects of the work.
Funding: This research was funded, conducted and approved by the Public Health Agency of Canada.

Data sharing: Data extracted from the included studies are presented largely in the Results and Appendices; however, full extraction data tables are available upon reasonable request from the corresponding author.

Acknowledgements: The authors thank the peer reviewers nominated by the Communicable and Infectious Disease Steering Committee (CIDSC) of the Pan-Canadian Public Health Network whose thoughtful comments and feedback on earlier versions of this evidence synthesis strengthened their work: Ian Davis, Riyas Fadel, Évelyne Fleury, Todd Hatchette, Laurie Ireland, Claude Laberge, Cynthia Lyles, David McKeown, Darrell Tan and Denise Werker. The authors also thank members of the CIDSC and the Council of Chief Medical Officers of Health of the Pan-Canadian Public Health Network for their reviews and comments. The authors acknowledge the advice provided by Dr. James Hanley with respect to our statistical analysis and interpretation. The authors also thank Jane Falconer for her external peer review of the search strategy for the update. We also acknowledge the unique contributions of our colleagues at the Public Health Agency of Canada, including Lisa Glandon, Lynda Gamble and Robyn Hocking at the Federal Science Library for preparing and conducting our electronic database searches; Fowsia Abdulkadir for assisting with reference screening; and Kanchana Amaratunga, Saskia Arnold, Marion Doull, Marsha Hay-Snyder, Howard Njoo, Toju Ogunremi, Dana Paquette, Shamila Shanmugasegaram, Winnie Siu and Karen Timmerman for their thoughtful input and insight.

Accepted: Sept. 20, 2018

Correspondence to: Rachel Rodin, rachel.rodin@canada.ca 